\title{
'It's led me astray': How Cognitive Behavioural Therapists experience personal therapy in clinical practice
}

Ariele Noble 1, $\bowtie$ Ariele Noble, PsychD. C.Psychol. is an HCPC-registered Counselling Psychologist Chartered with the British Psychologist Society. She is a Clinical Supervisor for Shout, as well as, a researcher in medical healthcare.

EmailAriele.Noble@mhiuk.org

Rosemary Rizq 2Rosemary Rizq, PhD. C.Psychol. AFBPsS. FHEA., is an HCPCregistered Counselling Psychologist Chartered with the British Psychological Society and a United Kingdom Council for Psychotherapy-accredited psychoanalytic psychotherapist. She is Professor of Psychoanalytic Psychotherapy at the University of Roehampton and worked for many years as Specialist Lead for Research and Development for National Health Service (NHS) Ealing. More recently, she worked in a specialist psychoanalytic psychotherapy service at North-East London NHS Foundation Trust's Forest House Clinic. She now has a part-time private practice in West London. Rosemary was submissions editor for Psychodynamic Practice from 2004 to 2010, is a reviewer for a number of academic journals and she has published widely on issues related to organisational dynamics and psychotherapeutic training and practice.

1Mental Health Innovations London UK

2Department of Psychology University of Roehampton London UK

Correspondence

Ariele Noble, Mental Health Innovations, 13 Bramley Road London W10 6SZ, UK.

Email: Ariele.Noble@mhiuk.org 


\section{Abstract}

Personal therapy is considered to be an essential component of most psychotherapeutic training programmes. However, it remains peripheral to cognitive behavioural therapy (CBT) training courses. We present a subsection of results from a qualitative study that examines how CBT therapists use personal therapy in their clinical practice. Seven CBT therapists who have undergone personal therapy were interviewed. Participants were asked about how they used personal therapy in their clinical practice and their accounts were analysed using interpretative phenomenological analysis (IPA) to identify common themes. This article presents the results of the first master theme, 'Personal therapy creates conflict', which explores a paradox that arises between personal therapy and CBT clinical practice; participants suggest that personal therapy equips them with therapeutic tools that paradoxically hinder their capacity to practice a standardised protocol-led CBT. Results show that participants found personal therapy created considerable internal conflict, where their use of technical evidence-based treatment protocols as practitioners was experienced in tension with the relationally oriented therapy they had received as clients. We discuss results in the context of Gabriel Marcel's philosophical insights on the dehumanising effects of technology on human relationships. We conclude with a brief consideration of the current political climate that increasingly privileges short-term technical solutions to psychological distress.

\section{Received: 12 February 2019 | Revised: 02 July 2019 | Accepted: 03 July 2019}

\section{Keywords}

clinical practice; cognitive behavioural therapy; interpretative phenomenological analysis; personal therapy; psychotherapy; therapist

\section{INTRODUCTION AND BACKGROUND TO STUDY}

Personal therapy for psychotherapists has been a focus of consideration over a century. Freud (1912/1958) first proposed personal therapy as an integral element of professional development amongst psychoanalysts. This has gradually developed into a controversy over whether the use of personal therapy amongst all psychotherapists is beneficial or even necessary (McNamara, 1986). 
An overwhelming body of research indicates that personal therapy is beneficial to clinical practice. Psychotherapists of all theoretical orientations who have had personal therapy find that it enhances professional and personal development and relational capacities (Farrell, 1996; Geller, Norcross, \& Orlinsky, 2005). The literature further suggests that personal therapy seems to enhance aspects of clinical practice that strengthen the therapeutic relationship: the clinician's self-awareness of personal issues, values, conflicts and defence mechanisms; increased empathy and interpersonal relatedness; and decreased chances of burnout and unethical behaviour (Macran \& Shapiro, 1998; Orlinsky, Schofield, Schroder, \& Kazantzis, 2011; Rizq \& Target, 2008a, 2008b, 2010).

A strong therapeutic relationship is a key factor in predicting positive outcomes. The significance of the therapeutic relationship on the outcome of psychotherapy dates back to Freud (1912/1958) and, similar to the topic of personal therapy, has become one of the oldest themes in therapy research. Initially derived from psychoanalysis, the concept of the therapeutic relationship has since been applied to all psychotherapeutic approaches and is deemed to be a significant indicator of therapeutic outcome (Bordin, 1979; Gelso \& Carter, 1985).

It is long accepted that, due to the client's heightened vulnerability, the therapeutic relationship needs to tolerate and withstand moments of misunderstanding and volatility (Bellah, Madsen, Sullivan, Swidler, \& Tipton, 2007). Personal therapy can help psychotherapists develop, refine and maintain the interpersonal skills they need to foster strong therapeutic relationships with their clients (Orlinsky, Geller, \& Norcross, 2005). Most psychotherapeutic training courses include mandatory personal therapy requirements. Yet the proponents of different theoretical models assign various values and purposes to personal therapy (Malikiosi-Loizos, 2013). Within the United Kingdom Council for Psychotherapy (UKCP), all 80 member organisations have extensive personal therapy training requirements, except for one: members of behavioural and cognitive psychology.

Cognitive behavioural therapy (CBT) is the only evidence-based psychotherapy intervention recommended for both depressive mood and anxiety disorders and, therefore, has become the most commonly provided treatment within the United Kingdom's National Health Service (NHS). Whilst personal therapy is formally encouraged in most counselling trainings, it is not formally encouraged in CBT. The CBT model's traditional focus on specific therapeutic techniques to promote psychological change serves to downplay the role of the CBT therapist's subjective perspective (Rizq, 2010). In this paper, we aim to add to the literature 
on the outcome of personal therapy for psychotherapists by exploring the perspective of CBT therapists, an over-represented yet under-researched group.

\section{RATIONALE FOR THE CURRENT STUDY}

The behavioural origins of CBT and the philosophy of science upon which it is based significantly influence its focus on the reduction of problematic cognitions and behaviours via well-defined and validated scientific techniques (Beck, 1967). The arrival of cognitivism in the 1960s joined the behavioural movement and placed the study of human learning and cognitive knowledge at the centre of empirical psychological research. The study of 'automatic negative thoughts' (Beck, 1967) identifies how certain errors of cognition and maladaptive reaction patterns can be changed (Beck, 1993).

CBT was initially established as a form of psycho-education, where therapists teach clients techniques to change attitudes, behaviours and cognitions (Malikiosi-Loizos, 2013). Its traditional focus on correcting irrational beliefs and faulty reasoning that are believed to underlie symptomology appears to preclude the need for therapists to engage in their own therapy, particularly if they are not experiencing any overt distress (Orlinsky et al., 2005). In addition, the efficacy of CBT is said to depend on technical adherence to uniform protocols. These are equally effective whether administered in a group or individual formats (Miller \& Berman, 1983). Indeed, Beck (1967) originally claimed that the success of CBT was due to its manualisation and the replication of its techniques by other therapists: "The same therapeutic program used by different therapists does not differ substantially from one to the other" (p. 333).

CBT's privileging of technical adherence and the empirical testing and verification of experience contrasts with more relational views of therapy that include notions of intersubjectivity and the therapeutic relationship (Orlans \& van Scoyoc, 2009). Sreenan (2013) finds that, "In general, studies tend to equate competence with technical skills and adherence to specific models rather than a broader sense of the word" (i.e., relational competence, emotional competence, etc.) (p. 44). Technical adherence is easier to monitor or rate than other more nuanced aspects of competence. Within the positivist approach to psychological interventions, the therapist's subjective perspective offers a variability in outcomes (Kim, Wampold, \& Bolt, 2006), which treatment protocols and training within CBT attempt to reduce. 
Over the last decade however, CBT researchers have started to investigate the emotional and interpersonal experiences of CBT therapists (Haarhoff, 2006) and to identify skills that support the therapeutic relationship such as empathy and working with difficult feelings towards clients (Bennett-Levy \& Thwaites, 2007; Malikiosi-Loizos, 2013). Indeed, it is now recognised that CBT demands a high degree of self-awareness (Orlinsky et al., 2005) especially when working with clients with long-standing, complex problems (Beck \& Freeman, 1990). Beck and Butler (2005) encourage personal therapy as an appropriate method of reflection within CBT clinical practice. Although personal therapy does not have a lengthy tradition in CBT, it is becoming accepted as a helpful way to improve clinical practice (Geller et al., 2005).

The literature on therapist's personal therapy has consistently demonstrated the difficulties of establishing whether personal therapy leads to better clinical outcomes (Macran \& Shapiro, 1998). Although most therapists rate the impact of personal therapy on their practice highly (Malikiosi-Loizos, 2013), objective evidence of its effectiveness remains weak (Bennett-Levy, Lee, Travers, Pohlman, \& Hamernik, 2003).

Given the difficulty of establishing a linear relationship between personal therapy and clinical outcomes, there is increasing interest in how therapists themselves understand the impact of personal therapy on their clinical work (Macran \& Shapiro, 1998). Rizq (2011) confirms that "in the last decade there has been a shift in interest from whether to how personal therapy influences client work" (p. 177), a shift that is reflected in recent research studies (Rizq \& Target, 2008a, 2008b). The epistemological swing from quantitative to qualitative methodologies, from objective outcome measures to subjective understanding of individual experiences, increasingly reflects researchers' interest in the subjective and individualised nature of the therapist's own perspective, and the collaborative therapeutic relationship deemed central to CBT clinical practice. This study, therefore, adopts a qualitative approach in exploring the perspective of CBT therapists on the relevance of personal therapy in clinical practice.

\section{METHODOLOGY}

\subsection{Study design}


Given the complexity and richness of the experience of personal therapy, we asked CBT therapists for in-depth descriptions of whether and how they experience and make meaning of personal therapy in their clinical practice. Interpretative phenomenological analysis (IPA) is a qualitative research method that facilitates access to personal meaning and sensemaking amongst individuals who share an experience (Smith, Flowers, \& Larkin, 2009). This epistemological stance privileges the careful interpretation of the individual's experience (Smith, 1996).

IPA involves two components: phenomenology and interpretation. Smith (2011) explains that IPA is "phenomenological in its concern with lived experience and... interpretative in recognising the analysis of experience as a hermeneutic activity" (p. 6). In other words, phenomenology seeks meaning, meaning is difficult to grasp, and so interpretation is necessary (Josselson, 2004). This method of research pursues "what the data means, not what it is" (Chamberlain, 2011, p. 52).

In our study, we attempted to understand and describe the meaning behind participants' experiences, to develop an interpretative analysis of participants' personal meaning-making and to provide a critical and conceptual commentary on the meaning behind participants' experiences (Smith \& Osborn, 2015). This paper explores what it meant for participants to have expressed feelings and concerns regarding their use of personal therapy in CBT clinical practice (Larkin, Watts, \& Clifton, 2006).

The complete study, undertaken as part of a doctoral research project, yielded three master themes and 12 subthemes presented below:

\begin{tabular}{|l|l|l|}
\hline Personal therapy creates conflict & $\begin{array}{l}\text { Personal therapy ties me to } \\
\text { humanity }\end{array}$ & $\begin{array}{l}\text { Personal therapy: Being and } \\
\text { Doing }\end{array}$ \\
\hline $\begin{array}{l}\text { Working in the shadow of what } \\
\text { CBT should be }\end{array}$ & Manifesting empathy & $\begin{array}{l}\text { Making room for emotional } \\
\text { experiencing }\end{array}$ \\
\hline Protocol versus Exploration & Recognising the client within & Being present \\
\hline Practice versus Preach & $\begin{array}{l}\text { Holding both positions as } \\
\text { therapist and client }\end{array}$ & $\begin{array}{l}\text { Participating in } \\
\text { therapeutic process }\end{array}$ \\
\hline Self and Other & Maintaining a space within & Seeking balance \\
\hline
\end{tabular}


For reasons of space, we present the results and analysis of only the first master theme: 'Personal therapy creates conflict'. While an overwhelming body of research suggests therapists generally find personal therapy to be useful, this theme from the study suggests that the outcome of personal therapy may be less straightforward for CBT therapists than for therapists trained in other modalities. Indeed, the theme highlights an internal conflict that appeared to be problematic for all participants. We were curious to articulate and clarify the nature of this conflict.

\subsection{Selection and recruitment of participants}

Qualitative research, and IPA specifically, requires data to be collected from an informationrich, rather than representative, participant pool (Rizq \& Target, 2008b). A homogeneous and purposive sample was recruited; a closely defined group for whom the research question was significant (Smith, 2015). The group of interest were accredited CBT therapists with at least five years of post-accreditation clinical experience and voluntary experience of weekly personal therapy for a minimum of two years. Recruitment included the following: emails to potential applicants listed on public registers; contact with university lecturers; and via a process of chain referral. Overall, seven individuals agreed to participate in the study and interviews took place over a seven-month period.

\subsection{Sample characteristics}

Three men and four women took part, with ages ranging from 35 to 71 . Four of the participants had trained via courses accredited by the British Psychological Society, two were qualified by the British Association of Counselling and Psychotherapy, and one via the UK Council for Psychotherapy. Four of the participants were accredited by the British Association for Behavioural and Cognitive Psychotherapy, two had Advanced Certifications in CBT and one had a Postgraduate Diploma in CBT. Post-accreditation clinical experience ranged from nine to 22 years. All were currently in clinical practice. Some participants may have had therapy as part of their training; however, the additional voluntary therapy was the basis of their selection. The length of time participants spent in voluntary personal therapy ranged from four to 15 years. Theoretical orientations of personal therapy varied. Three participants identified the theoretical orientation of their personal therapy as Integrative; one as Pluralistic/Humanistic; one as Psychoanalytic/Systemic; one as Jungian; and one participant had both experiences of Psychodynamic psychotherapy and CBT. 


\subsection{Data collection: interview procedures}

Due to the high level of sensitivity necessary in dealing with the confidential and personal nature of this research topic, in-depth, semi-structured interviewing was the most effective method for data collection (Rizq \& Target, 2010; Smith, 1996). Participants were informed about the aims of the research and were given the opportunity to ask questions throughout the interview process.

To maintain the openness required for in-depth exploration, the researcher incorporated interview guidelines offered by Smith (2015) and Smith et al. (2009) into the design of the interview. The questions for the interview schedule were developed by the researcher based on personal experience of personal therapy and clinical practice, discussions with colleagues and from reading the existing literature on personal therapy and CBT. The interview schedule included questions such as: What led you to undertake personal therapy? Do you feel that personal therapy has influenced your CBT clinical practice; how or how not; what are some examples? Can you think of a time in clinical practice when you were aware of your personal therapy; in what way? Do you feel that your personal therapy has been influential in maintaining effective therapeutic relationships; if so, how; can you give examples?

Interviews lasted between one and one and a half hours and took place in a conveniently located, comfortable and private setting of participants' choosing. Interviews departed from the interview schedule guided by participants' contributions (Smith \& Osborn, 2015). Interviews were transcribed verbatim.

\subsection{Analysis and validity}

Following the analytic approach for IPA outlined by Smith et al. (2009), the preliminary analysis involved the analysis of each individual transcript as if it were the only piece of data. To promote the idiographic perspective, each transcript was read and re-read in detail alongside listening to the audio-recording. In line with IPA, the researcher's interpretation of the text was a necessary element in capturing the meaning behind participants' experiences (Josselson, 2004; Smith, 1996). Significant issues, topics, ideas, and feelings were noted by the researcher along the transcript margin. These margin notes were used to create clusters of similar topics and issues that developed into themes and subthemes. A list of themes and 
subthemes were generated for each transcript, anchored by extracts that conveyed an essential expression of each theme (Dickson, Knussen, \& Flowers, 2008). This analytic process was manually repeated for each individual transcript as if it were the only piece of data collected.

To promote transparency (Yardley, 2008) and validity (Stiles, 1993) in the emerging analysis, each participant received their anonymised interview transcript and individual analysis to explore whether they recognised themselves in the researcher's interpretations. Three of the seven participants provided feedback. Once participant feedback was collected, a cross-case comparison of the individual analyses was manually conducted to search for patterns across the analyses (Smith, 2011). When similar patterns emerged from at least half of the individual analyses, they were identified as recurrent (Dickson et al., 2008). Recurrent themes and subthemes were organised into a table of master themes. Like the individual analyses described above, the cross-case analysis was written in narrative form anchored by extracts that conveyed an essential expression of each theme.

\section{Results}

The results of our study were in some ways different to what we might have expected from the literature. An overwhelming body of research suggests that psychotherapists of all theoretical orientations who have had personal therapy find it professionally useful (Farrell, 1996; Geller et al., 2005). Yet results from this study suggest that the process of personal therapy might be more complex for CBT therapists.

The aspects of personal therapy participants had found helpful to their clinical practice conflicted with their identity as CBT therapists. For example, participants similarly valued a process of 'exploration' gained from their personal therapy, however, also seemed to share the concern that this very process they valued conflicted with the priorities of adhering to protocols that were expected of them. Although relational aspects of therapy are increasingly acknowledged as key to positive therapeutic outcomes (Orlinsky et al., 2005), traditions of privileging technical adherence often portray relational aspects as secondary (Sreenan, 2013).

All participants appeared to incorporate relational aspects of therapy into their clinical practice in ways that seemed at least as important as technical adherence to protocol. Yet it was in these moments that participants also appeared to struggle against a shared notion 
that prioritises adherence to protocol. The aspects of personal therapy participants found useful to their clinical practice paradoxically led them 'astray' from adhering to protocol.

\subsection{Master theme: Personal therapy creates conflict}

\subsubsection{Subtheme 1: Working in the shadow of what CBT should be}

All participants recounted experiences of delivering something more, or different, to 'pure CBT' protocol in their clinical practice. This seemed to result in a sense of tension. Whilst participants all spoke about the importance of adhering to strict CBT protocols, they also spoke about the way in which personal therapy had equipped them with therapeutic tools that paradoxically hindered their capacity to do so. It was clear that all participants felt they benefited from their personal therapy, both personally and professionally, yet also seemed to share the sense of being 'led astray' by it.

Roth and Pilling (2008) indicate a comparable gap between the protocol-based priorities of CBT as a science and as an art. Their 'competence framework' incorporates 'metacompetencies' for a better evidence-based intervention. 'Metacompetencies' are skills to apply the science-based therapy artfully: in a flexible and individually tailored way. Participants seemed to suggest that, while personal therapy equipped them with the skills to practice CBT 'artfully', this came at a certain cost.

For example, Dorinda conveys her sense of deviating from 'pure CBT protocol' with what she has 'gained' from personal therapy. Yet her use of contradictions, 'completely almost' and 'really influenced by just a little tiny bit', illustrates tension around incorporating relational aspects into her clinical practice:

It's completely almost pure CBT protocol but really influenced by just a little tiny bit of psychodynamic thinking that, sort of, I gained from looking at my own processes, looking at my own therapy, what I re- enact from the past.

Peter similarly discusses how, as a result of his personal therapy, he has gained the ability to depend less on CBT protocols and, instead, uses a CBT framework as a more flexible guideline from which to follow the client's lead. Peter's sense of being 'led astray' seems to illustrate a conflict between his clinical practice and 'a rigid CBT point of view' from which he deviates: 
For me protocols now - as a result of the, uh, personal therapy, protocols are a guide, but they're not a must...I'm quite capable of deviating slightly... So, you could say, from a rigid CBT point of view, it's led me astray.

Raul, too, distinguishes his clinical practice from a CBT approach based on a 'formula' of 'targets' and 'goals'. His clinical focus to 'help people understand who they are ' seems to conflict with the image of a more 'formulaic' CBT:

But that's really working to $a$, to the formula....and if that's your target and that's your goal, which is absolutely legitimate and nothing, nothing wrong with it...then that's what you do but...it's not, it doesn't have the same components that the work that I would seek to do, which is to help people understand who they are.

Participants seemed to describe their clinical practice against the backdrop of a CBT 'norm' that privileges technical adherence. It was as if they held their own way of practicing CBT in a constant state of tension stemming from a shared notion that the relational influence of their personal therapy in their clinical practice was second to adhering to protocol. Bennett-Levy, Thwaites, Chaddock, and Davis (2009) support that, amongst CBT practitioners, personal therapy is typically a long and deep process without necessarily having significant clinical implications. Yet participants in our study reported reaping significant clinical gains from their personal therapy and, moreover, viewed the capacity to deviate from CBT protocol as an achievement.

\subsubsection{Subtheme 2: Protocol versus Exploration}

All participants distinguished between a personal experience of therapy as a journey of exploration and discovery and the somewhat different experience of delivering a protocol-led CBT to their clients. The long and difficult process of personal change and development experienced and valued by participants seemed to conflict with the pre-determined code of procedures prioritised by the CBT model they practiced. Five out of the seven participants appeared to illustrate their struggle for equality between their relational priorities and the privileging of technical adherence in their clinical practice. Participants seemed to believe 
that personal therapy influenced their clinical practice in ways that were at least as important as the adherence to protocols expected of them as CBT therapists.

For example, Peter spoke about how he struggled with his protocol-driven CBT training. Below he describes his early 'treatment failures' as relying too heavily on CBT protocols. Like a machine, his capacity to subjectively engage with clients was hindered:

Certainly, some of my, inverted commas, treatment failures, I've actually been too heavy on a, the sort of delivery protocol...I appear times mechanistic, and I've sometimes thought, oh god I didn't really relate to that person at all.

Dorinda similarly discusses her sense of responsibility to follow CBT protocols and how the space for a deeper, less content-driven therapeutic exploration of feelings she values seems less important. Here, she describes her experience with clients who appear to hand over their problems for her to manage. She likens her role to that of a 'medical doctor', which implies an objective, content-driven, problem-solving approach to treatment. In ironically aligning herself with a medical doctor, Dorinda suggests that 'CBT doesn't have space' for self-awareness, uncertainty, and anxiety:

They come in, and they go woosh 'Here's all my stuff, you deal with it'. And then you kind of diagnose and you treat. So very medical model. Doctor my foot is hurting. But I'm much more aware that... there is a whole host of anxieties before you even enter the therapy room...that CBT doesn't have space for.

Charlotte, too, describes her own experience of feeling encumbered by the CBT model's protocol-driven priorities. It is almost as if Charlotte finds that the CBT model hinders her ability to connect with her client's emotional distress. She struggles with what appears to be a choice between adhering to CBT protocol or listening more deeply to her client and allowing more space for 'her pain':

If I continued with my structured sort of way... I would've completely overlooked [my client's] needs and what was so alive in the room, which was she wanted me to sit and allow her to be and for me to actually hear what-her pain. 
Participants' distinct experiences of personal therapy and clinical practice seemed to reveal a greater distinction between the personal therapy they received and the therapy they practiced and between the respective functions of therapy as a journey of exploration and discovery and of therapy as a delivery of problem-solving protocols. Laireiter (2000) finds that a majority of CBT therapists who undergo personal therapy prefer an alternative approach, compared to about 15 percent who prefer to undergo CBT. All the participants of this study preferred to undergo therapy of an alternative approach to their clinical practice. Six out of the seven participants spoke about the differences between their personal therapy and clinical practice.

\subsubsection{Subtheme 3: Practice versus Preach}

There appeared to be a particular tension for participants between therapy that aims to solve a particular problem and one that aims to enhance growth and development. Participants described their personal therapy as a way 'to better myself', 'enhance wellbeing', and 'to improve my life'. Participants who thought of therapy as a way to better themselves simultaneously understood the therapy they offered as a 'goal-driven' 'crisis intervention' meant to help overcome the sense of self-defeat. This striking difference between the therapy participants sought and the therapy they delivered appeared to illustrate a fundamental divergence of attitude between undergoing therapy due to a desire for self-improvement and undergoing therapy because of an urgent sense of need or desperation.

For example, Dorinda identifies CBT as a professional intervention 'fantastic at a crisis' to help people in urgent need. In contrast, Dorinda appears to seek personal therapy for her own personal desire to enhance 'myself':

I don't go to therapy for a point of, 'My life is not working out and I need help'. So, I don't go to therapy from a sort of mental health crisis point of view. I go to therapy as a way of self-discovery. Making myself, eh, just a better person for myself.

Similarly, Raul differentiates between his personal therapy as a means of enrichment and his belief in the more common approach to CBT as a means to overcome a specific 
problem. Furthermore, it seems that Raul's portrayal of life as 'traumatic' serves to normalise his more casual use of therapy:

I'm in personal therapy for richness...I'm not working on any particular um, uh, traumatic issue apart from life.

Hank more overtly explains his use of a less goal-driven personal therapy as a means to counterbalance the goal-driven nature of his clinical practice. In this way, personal therapy appears to offer Hank a sense of balance:

The payoff is I'm goal driven in my sessions but then when I go to my own personal therapy it allows me to just, just be...but with my, with the client base that I have it's not about just being, it's about working towards the goal. And that's why they come to see a cognitive therapist rather than someone else.

The difference between the therapy participants sought and the therapy they delivered seemed to elicit a sense of distance between themselves as clients and their clients. Wilson, Weatherhead, and Davies (2015) explores the personal therapy experiences of trainee clinical psychologists predominantly trained in CBT and indicates the presence of stigma: personal therapy is a weakness and therapists are expected to be 'more sorted' (p. 11). However, participants of this study appeared to celebrate their roles as clients as a way to align themselves with their clients.

Participants' use of their own personal therapy appeared different from their clients' use of CBT. Yet as therapists, participants' experiences of personal therapy modelled the flexibility and openness that seemed to enhance their capacity to connect with, and relate to, their clients' unique qualities, bridging the gap between self and other.

\subsubsection{Subtheme 4: Self and Other}

The more participants learned about themselves the more they seemed to allow themselves to relate to their clients. Participants appeared to use their personal therapy to become aware of their own feelings and to share themselves as a necessary and useful part of their clinical practice. Personal therapy seemed to facilitate participants' capacities to assume a clinical responsibility to think about what had gone on between them and their clients. All of 
the participants conveyed that their own self-awareness allowed them to engage with and connect emotionally with their clients. The following extracts illustrate participants' capacities for reflexivity and illuminate a fundamental relationship between self and other in clinical practice.

For example, Charlotte recounts the first time she became aware of her potential influence on her client. Her experience seems to illustrate a shift from a clinically objective process by which she keeps herself out of, or separate from, her client's experience, to a subjective process by which she considers and shares herself as part of her client's experience:

I started thinking about, um, the feelings that are being, that are experienced in a room; how much of it is the client's, how much of it mine.

Hank similarly recounts his own experience of becoming aware of his feelings in relation to his client and describes what appears to be a confusion between his own and his client' s 'anxiety':

I took on [my client's] anxiety, and I brought that to therapy to sort of look at that, that I had her anxiety...that's where therapy will help or help me sort of move forward, um, where I can just go, okay let's just pull the bones out of something.

Karly takes a step further by discussing how she uses her self-awareness to inform her therapeutic interventions. In the following example, she describes how difficult-and yet how necessary-it is to know how she feels in order to respond thoughtfully to her client. Karly's openness to the choices available to her seems relevant to the topic of subjective engagement discussed earlier in the subtheme, 'Protocol versus Exploration', with Peter. Like Peter, Karly's prime concern seems to be about how she relates to her clients and appears to demonstrate her clinical capacity to take responsibility for her own motivations:

I'll think, what's my motivation? Am I trying to prove that I'm a good therapist? In that moment, what's going on for me? Am I feeling vulnerable? If I'm feeling vulnerable, is it my vulnerability? Is it their vulnerability? So, all of that has to happen really quickly as a therapist in your, in those nanoseconds when it whizzes through 
your brain. And then it's within, it becomes a choice as to whether I then make an informed intervention.

Personal therapy appeared to conflict with many aspects of the protocol-driven CBT model as perceived by participants. Yet the very aspects that conflicted with their identity as CBT therapists seemed to inversely tie them closer together with their clients. Personal therapy appeared to facilitate a space for individuality and flexibility fundamental to participants' clinical practice. Their capacities to 'use the self' to take responsibility for their own feelings and vulnerabilities seemed to strengthen participants' capacities to tolerate the feelings and vulnerabilities of their clients.

\section{DISCUSSION}

We feel it is significant that all participants spoke about how their personal therapy seemed to create a sense of conflict within their clinical work. All participants felt they benefited from their personal therapy, both personally and professionally, yet also seemed to share the sense of being 'led astray' by it. It is now widely recognised that CBT demands a high degree of self-awareness (Orlinsky et al., 2005) and that personal therapy is a helpful way to improve CBT clinical practice (Geller et al., 2005). Yet CBT's tradition of privileging technical adherence continues to conflict with more relational views of therapy, such as intersubjectivity and the therapeutic relationship (Orlans \& van Scoyoc, 2009). Relational and emotional competencies often fall second to technical competence and protocol adherence (Sreenan, 2013). Yet personal therapy was essential to participants' clinical practices in ways that were at least as important as technical competence and protocol adherence.

Participants' experiences of personal therapy modelled the flexibility and openness that seemed to enhance their capacity to connect with, and relate to, their clients. Their ability to deviate from evidence-based protocol in this way was seen as an achievement attributed to the long and difficult process of personal change and development participants experienced and valued. However, these relational achievements in their clinical practice created conflict with their identity as CBT therapists.

It was precisely those aspects of personal therapy that participants spoke of as most helpful that appeared to be at odds with their professional identity. For example, Dorinda felt she 
had to deviate from 'pure CBT' to incorporate 'what I re-enact from the past'. Charlotte, too, chose to stray from 'structured' CBT to listen more deeply and 'actually hear [her client's] pain'. Similarly, it was moments of strict protocol adherence where Peter felt 'mechanistic' and unable to 'relate' to clients. It seemed that in moments of connecting with, and relating to, their clients, participants shared a sense of being 'led astray'.

Participants' experiences of conflict between their personal therapy and clinical practice can be seen to reflect a dilemma in the field of psychology in which the prevailing political culture seems to dismiss the therapist's 'use of self' in place of 'pure' and ready-made solutions, prioritising adherence to treatment strategies over the relational components of clinical theory and practice. We have chosen here to draw on Gabriel Marcel's existential theories $(1949,1952,1963,1964)$ that we suggest may offer helpful insights into the dehumanising effects of contemporary technologies.

Marcel (1952) was critical of science for its dehumanising effects, as well as its focus on solving problems. A 'problem' is a question that is meant to be answered objectively and in which, consequently, the identity of the questioner becomes irrelevant. In contrast, a 'mystery' represents a process of exploration and meaning-making, which, consequently, invites the questioner to subjectively participate, facilitate and be touched by 'the other' experience (Marcel, 1963). Once the object of a problem is understood or solved, it is considered complete, whereas the subject of a mystery always involves the other and so always remains open, alive, and interesting (Marcel, 1949).

Participants highlight a major thread of Marcel's (1952) philosophy that raises the issue of protecting one's subjectivity from annihilation within a technology-driven society. Participants spoke about how their personal therapy equipped them with therapeutic tools that paradoxically hindered their capacity to strictly adhere to CBT protocols. This tension seems to stem from the rise of treatment protocols within the field of psychology. Although relational aspects of clinical practice are widely acknowledged as a significant indicator of therapeutic outcome (Bennett-Levy \& Thwaites, 2007), the space for one's own individuality as a therapist too easily falls second to techniques and protocols.

This study indicates that what one gains from personal therapy in clinical practice can be at least as important as protocol adherence. The value of exploring clients' mysteries should be at least as important as the value of human problems being solved similarly from person to person with a technique that, arguably, could be employed by anyone (Treanor \& 
Sweetman, 2016). As Dorinda recounts: "They come in, and they go woosh 'Here's all my stuff, you deal with it'. And then you kind of diagnose and you treat...Doctor my foot is hurting". In speaking about her clinical practice, Dorinda raises the issue of becoming a problem-solver.

Evidence-based practice emerged in psychology to improve clinical outcomes by getting clinicians to base their choice of interventions on empirical evidence rather than clinical impression, intuition and convention (Sackett \& Rosenberg, 1995). This study highlights the risk of adopting a 'technological mindset' within protocol-based interventions (Marcel, 1952), qualifying ourselves and others in terms of the various functions we perform and the evidence we produce, almost like a machine. Viewing psychological distress as a 'problem' to be solved risks disregarding the mysterious worth at the centre of each human being which cannot be easily summed up or defined (Marcel, 1952). However, exploring a 'mystery' requires the therapist's personal involvement and investment in the therapeutic process that risks their vulnerability.

Our findings demonstrate how personal therapy can serve to enhance self-reflection, to familiarise oneself with one's own vulnerabilities, and to facilitate one's capacities to share oneself with one's clients in order to be a part of one's clients' clinical experiences. Indeed, accounts suggest that personal therapy can facilitate a space for the individuality and flexibility fundamental to empathic and effective clinical practice. The use of the self as a means of the therapist taking responsibility for their own feelings and vulnerabilities appears to strengthen their capacity to tolerate the feelings and vulnerabilities of their clients.

\subsection{Limitations}

For reasons of space, we present an abbreviated version of only the first master theme of the complete study, which limits the representation of each participant and reduces from the already small sample. A small sample can nonetheless serve to maximise results by highlighting significant, and otherwise unnoticed, phenomena (Larkin et al., 2006). IPA was chosen as an appropriate methodology for this study given the research aim to provide a rich insight into the lived experience of therapists using personal therapy in CBT clinical practice. IPA does not seek generalisable findings but could rather be said to shed light on the universal by looking in detail at the particular (Warnock, 1987). 
It is important to note that all participants of this study reported positive experiences of personal therapy. This research may have elicited this particular sample of therapists on the basis of the researchers' beliefs and assumptions. Even when closely monitored, researchers hold an agenda that can limit and steer the recruitment and interview processes. Our pre-existing beliefs and assumptions about the positive benefits and value of personal therapy might have been indicated in our language, influencing interviews in a direction the participant might not have chosen otherwise (Potter \& Hepburn, 2005). In light of the overwhelmingly positive experiences of personal therapy in this study, a useful subsequent study could explore the experiences of CBT therapists who have found personal therapy unhelpful or irrelevant to their clinical practice.

\section{CONCLUSION}

The uniform protocols advocated by CBT appear to underpin its reputed efficacy. However, participants in our study all appeared to struggle with the tension between adhering to treatment protocols and using their own subjective perspectives to guide therapeutic work. Dismissing the therapist's 'use of self' can serve to reduce CBT to a collection of techniques. To paraphrase Marcel (1964), guiding the client through a form or formula runs the risk of the therapist being no more useful than a pen. The impersonal nature seems to imply that both parties are detached and replaceable (Marcel, 1964).

This study illuminates a split in the psychotherapy profession between protocol- and relational-based practices, and the tensions that arise from attempting to balance its deepening contradictions. We caution that the prevailing political culture has become strongly influenced by the seductiveness of automated ready-made solutions and compromises the credibility of individuality and of human connection in mental health care and treatment. This study indicates that the 'use of self' and being vulnerable to one's clients are at least as important as 'technical' and prescriptive models of therapy. Indeed, results suggest that the relational gains made in personal therapy may paradoxically make it more difficult for therapists to adhere to protocols that are generally assumed to significantly improve clinical outcomes in CBT.

The development of the government's Improving Access to Psychological Therapies (IAPT) programme that specifically privileges short-term CBT is part and parcel of a cultural shift that endorses technical solutions to problems of mental and emotional distress. The development of manualised protocols and computerised CBT is now occurring within a 
socio-political context that insists on improved clinical outcomes at as low a cost as possible (Layard, Bell, \& Clarke, 2006). The rising demand for talking therapies together with a lack of trained therapists means there is increasing pressure to develop digital technologies and mental health 'apps' whose popularity now challenges the significance of relational forms of practice deemed central to traditional therapeutic training (Reger \& Gahm, 2009).

Our participants demonstrate that the therapist's 'use of self' can conflict with the priorities of protocol adherence. We suggest that this tension can undermine the humanity of the therapist and the already difficult job of connecting with and being deeply affected by the other. We argue that personal therapy may help therapists to navigate the difficulties in being vulnerable and open to emotional engagement with their clients. Finally, we would like to suggest, along with Marcel, that personal therapy may enable us to think of clients as 'mysteries' to be encountered rather than as 'problems' to be solved. 